\title{
Authors' reply to Schaffalitzky de Muckadell and colleague's Comment on "Evaluation of the Reported Rates of Severe Hypersensitivity Reactions Associated with Ferric Carboxymaltose and Iron (III) Isomaltoside 1000 in Europe Based on Data from EudraVigilance and VigiBase ${ }^{\mathrm{TM}}$ between 2014 and 2017"
}

\author{
Birgit Ehlken ${ }^{1} \cdot$ Lennart Nathell $^{2} \cdot$ Annegret Gohlke $^{1} \cdot$ Derya Bocuk $^{1} \cdot$ Massoud Toussi $^{3} \cdot$ Stefan Wohlfeil $^{4}$
}

Published online: 1 April 2019

(c) The Author(s) 2019

\section{Dear Editor,}

We write in response to the letter by Schaffalitzky de Muckadell and Strom [1] on behalf of Pharmacosmos, the manufacturer of the iron (III) isomaltoside 1000 product. This letter contains a number of criticisms regarding the method used in our article Evaluation of the Reported Rates of Severe Hypersensitivity Reactions Associated with Ferric Carboxymaltose and Iron (III) Isomaltoside 1000 in Europe Based on Data from EudraVigilance and VigiBase ${ }^{\mathrm{TM}}$ between 2014 and 2017 [2]. We appreciate the interest the manufacturer, Pharmacosmos, has in the details of the published analysis. However, we take issue with and wholly disagree with the claim that the article was either incorrect, scientifically invalid or misleading. In the following, we address the specific criticisms that were articulated, none of which we believe alter the analysis or its findings, and in doing so, we reaffirm the methods and conclusions in our article.

1. Our article used a pharmaco-epidemiological methodology to assess reporting rates with respect to exposure,

This reply refers to the article available at https://doi.org/10.1007/ s40264-019-00814-5.

Annegret Gohlke

dr.annegret.gohlke@iqvia.com

IQVIA, Landshuter Allee 10, 80637 Munich, Germany

2 Nathell Medical AB, Växthusvägen 6, 58276 Linköping, Sweden

3 IQVIA, 17 Bis Place des Reflets, TSA 64567, 92099 La Défense Cedex, France

4 Vifor Pharma Management Ltd., Flughofstrasse 61, 8152 Glattbrugg, Switzerland using real-world data, including sales data to assess drug exposure. This methodology has been previously published [3] and is used in periodic safety update reports, which are highly valued by regulatory authorities. While the article very clearly articulates the potential limitations of methodologies that rely upon spontaneous adverse event (AE) reporting, such analyses do provide valuable insights when potential biases are anticipated and when the results are used together with other sources of data to form part of the overall mosaic of insights from prospective randomized controlled studies, epidemiological analyses, case studies, and the like. We agree with the intent of the European Medicines Agency (EMA) quote [4] that solely depending upon the number of reported AEs without considering exposure, known reporting biases or other known confounders, is inappropriate. Indeed, our article clearly states that "the presented results do not allow a conclusion to be drawn about the absolute and relative risk for severe hypersensitivity reactions (HSRs) associated with ferric carboxymaltose (FCM) and iron (III) isomaltoside 1000." Nevertheless, our analysis mitigates a number of known and potential biases, including using a uniform method of normalizing for patient exposure as well as a number of other potential biases such as the time trends in $\mathrm{AE}$ reporting, which we address in the article itself and subsequently in the following.

2. The letter by Schaffalitzky de Muckadell and Strom [1] suggests that the analysis period may have introduced a bias. This assumption refers to time trends in AE reporting, also called the "Weber effect," sometimes described as "AE reporting peaks at the end of the second year after a regulatory authority approves a drug" [5]. We discuss the impact of time trends in AE reporting in our 
article, and the study period was chosen to represent a time window where both products had been on the market for significantly more than 3 years. The most recent market event, namely the EMA referral on intravenous irons, would have affected both products simultaneously. Furthermore, it has been published that, after the first years of use, there is a tendency for only serious adverse drug reactions (ADRs) to be reported [6], and the article focused on severe HSRs in a period where both products were past their first years of use. Our analysis also provided reporting rate ratios by year. The last year of analysis (2017) — with a time difference of more than 4 years from the period the products were last launched in the majority of countries-also provided a higher reporting rate for iron (III) isomaltoside 1000 than for FCM.

The letter [1] presents a graph summarizing a calculation on Swissmedic data for FCM for the time period 20102013 [7] and VigiBase and EudraVigilance data for iron (III) isomaltoside 1000 for the period 2014-2017, reportedly taken from our article. However, it should be noted that the Swissmedic data have been generated via Standardized MedDRA ${ }^{\circledR}$ Questions, which have a wider definition for anaphylactic reactions, and is therefore not identical to the four MedDRA ${ }^{\circledR}$ preferred terms used in the article [2], which invalidates the comparison in the letter [1]. Furthermore, when applying the same consistent methodology to Swissmedic data, we find reporting rates for FCM similar to those we described in the article, namely a rate of 0.53 per 100,000 defined daily doses (DDDs) in the period 1 January 2010 to 31 December 2013 and 0.61 per 100,000 DDD in the period 1 January 2014 to 31 December 2017 (unpublished data), which speaks against any analysis period bias for severe hypersensitivity.

3. The letter [1] criticizes the restriction of the MedDRA ${ }^{\circledR}$ search to four terms. In fact, the objective and focus of this study was specifically to assess reporting rates for severe HSRs, as was clearly stated in the study. In contrast to clinical trial data, the use of real-world data introduces variability and heterogeneity, and-for this reason-a narrow definition was utilized to minimize other sources of heterogeneity with respect to anaphylactic/anaphylactoid reactions. For both studied products, anaphylactic/anaphylactoid reactions are specifically stated as a serious and potentially fatal reaction in the warning section (4.4) in the respective summaries of product characteristics. HSRs are generally serious adverse events but vary regarding severity grades. In order to focus on the more severe reactions with substantial consequences including fatality, we narrowed the search to severe reactions. The term "anaphylactoid/anaphylactic reactions" is also a specific term stated in the table of ADRs for both products. For those reasons, we defined severe hypersensitivity as anaphylactic reaction, anaphylactic shock, anaphylactoid reaction or anaphylactoid shock and used the specific MedDRA ${ }^{\circledR}$ preferred term for the extraction of data for both products.

Broadening the search terms does not necessarily reduce the risk for bias, as other factors such as reduced specificity of the search terms might become relevant. Thus, it is difficult to comment on the calculation presented in the letter for a broader definition of hypersensitivity events, as the underlying exposure data have not been presented in the letter, invalidating any comparison.

4. The letter [1] suggests, without providing quantitative reasoning, that the use of iron (III) isomaltoside 1000 would be underestimated. In fact, for both substances, 100, 500, and $1000 \mathrm{mg}$ vials are available and were included in the analysis. As presented in the article, analysis of administered doses that were reported in EudraVigilance did not reveal noticeable differences. The assumption in the letter, that inclusion of exposure to specific formulations of low-dose iron (III) isomaltoside 1000 results in overestimation of the reporting frequency of iron (III) isomaltoside 1000, can therefore not be substantiated.

The IQVIA MIDAS data were chosen to allow a consistent basis across geographies and products. We are aware that individual company sales data can differ from MIDAS because of individual manufacturer's sales reporting preferences, but a consistent database covering all products (and not specific to any one product or even class) is used to enable analyses unbiased by these effects. It should be noted that MIDAS data, due to this consistency and lack of company bias, are used widely in both market research and pharmacoepidemiology, for example by the World Health Organization (http://www. who.int and references therein).

5. The assertion in the letter [1] that relevant literature and clinical trial findings were ignored, is untrue. In fact, the available literature was discussed (e.g., quoting Aksan et al. [8], Bager et al. [9] and Mulder et al. [10]). However, randomized controlled trials and real-world data provide complementary perspectives, as acknowledged by the US FDA in their recent strategic framework [11].

Specifically, in this context, clinical trial settings are limited by the fact that anaphylactic/anaphylactoid reactions and shocks are fortunately very rare events. An extremely high number of subjects would be required to generate sufficient statistical power for these side effects in a trial. Given the rarity of the events, we analysed post-marketing spontaneous reporting of ADRs in the real world to base our analysis on a much larger sample size and exposure. 
With respect to the references named in the letter [1], it is difficult to see how they support the Pharmacosmos case; one reference compares iron (III) isomaltoside 1000 with iron sucrose and not with FCM [12]. Another is a meta-analysis and provides no head-to head data [13]. The results from the PHOSPHARE studies [14] comparing FCM and iron (III) isomaltoside 1000, are, to the best of our knowledge, not yet published in full in a peer-reviewed journal as this refers to an abstract that will be presented in March 2019. Hence, we cannot comment on how these studies would deal with the sample size challenge for rare events. To conclude, the referenced studies only confirm the need for further comparative studies on severe HSRs associated with FCM and iron (III) isomaltoside 1000, as stated in the discussion in our article.

The use of real-world data provides valuable information, which contributes unique insights that assist in developing a broader and more accurate assessment of the true nature of illness and the impact of therapies. The results of our analysis are consistent with other insights that we describe in our article.

While we are convinced about the validity of our study and its findings utilizing real-world data, we value different perspectives and are eager to engage in a spirited and evidence-based debate about the interpretation of the data and magnitude of effect that was demonstrated. However, we vigorously refute any assertion that impugns the motivations of the analysis that was performed. Its goal was to contribute relevant, valid insights based on accredited rigorous scientific methods, which would broaden the evidence for the benefit of patients. The article was clear that the study was funded by Vifor and that the IQVIA authors were part of the study team. While IQVIA received funding for the study, IQVIA employees receive their salaries from IQVIA; they received no remuneration from Vifor, and it is improper to suggest otherwise. Furthermore, to suggest that our work is a form of "concealed promotion" is wholly inaccurate and scurrilous and demeans the spirit of vigorous debate on which the peer-review and scientific method are based.

\section{Compliance with Ethical Standards}

Conflicts of interest Stefan Wohlfeil is an employee of Vifor Pharma Management Ltd. Lennart Nathell is a former employee of Vifor Pharma, and-during the planning, execution and publishing of the study - was working for a company on a contract with Vifor Pharma Management Ltd. Vifor Pharma is the manufacturer for Ferinject ${ }^{\circledR}$ (ferric carboxymaltose). Birgit Ehlken, Annegret Gohlke, Derya Bocuk and Massoud Toussi are employees of IQVIA, which received funding from Vifor Pharma International AG for the conduct of the study.

Funding This study was funded by Vifor Pharma International AG.
Open Access This article is distributed under the terms of the Creative Commons Attribution-NonCommercial 4.0 International License (http://creativecommons.org/licenses/by-nc/4.0/), which permits any noncommercial use, distribution, and reproduction in any medium, provided you give appropriate credit to the original author(s) and the source, provide a link to the Creative Commons license, and indicate if changes were made.

\section{References}

1. Schaffalitzky de Muckadell P, Strom CC. Comment on "Evaluation of the reported rates of severe hypersensitivity reactions associated with ferric carboxymaltose and iron (III) isomaltoside 1000 in Europe based on data from EudraVigilance and VigiBase ${ }^{\mathrm{TM}}$ between 2014 and 2017". Drug Saf. 2019. https://doi.org/10.1007/s4026 4-019-00814-5

2. Ehlken B, Nathell L, Gohlke A, Bocuk D, Toussi M, Wohlfeil S. Evaluation of the reported rates of severe hypersensitivity reactions associated with ferric carboxymaltose and iron (III) isomaltoside 1000 in Europe based on data from EudraVigilance and VigiBase ${ }^{\mathrm{TM}}$ between 2014 and 2017. Drug Saf. 2019;42(3):463-71. https://doi. org/10.1007/s40264-018-0769-5.

3. Bailie GR, Horl WH, Verhoef J-J. Differences in spontaneously reported hypersensitivity and serious adverse events for intravenous iron preparations: comparison of Europe and North America. Arzneimittelforschung. 2011;61:267-75. https://doi. org/10.1055/s-0031-1296198.

4. European Medicines Agency. Rapid response to BMJ. Re: Pandemrix vaccine: why was the public not told of early warning signs? (EMA/659264/2018). https://www.ema.europa.eu/documents/other leuropean-medicines-agency-rapid-response-british-medical-journ al-pandemrix_.pdf. Accessed 25 Mar 2019.

5. Hoffman KB, Dimbil M, Erdman CB, Tatonetti NP, Overstreet BM. The Weber effect and the United States Food and Drug Administration's Adverse Event Reporting System (FAERS): analysis of sixtytwo drugs approved from 2006 to 2010. Drug Saf. 2014;37:283-94. https://doi.org/10.1007/s40264-014-0150-2.

6. Moulis G, Sommet A, Durrieu G, Bagheri H, Lapeyre-Mestre M, Montastruc J-L. Trends of reporting of 'serious' vs. 'non-serious' adverse drug reactions over time: a study in the French PharmacoVigilance Database. Br J Clin Pharmacol. 2012;74:201-4. https ://doi.org/10.1111/j.1365-2125.2012.04185.x.

7. Swissmedic. Vigilance-news. Edition No. 11. December 2013. https:// www.swissmedic.ch/dam/swissmedic/en/dokumente/marktueber wachung/vigilance/vigilance-news_editionno11-dezember2013.pdf. download.pdf/vigilance-news_editionno11-december2013.pdf. Accessed 21 Feb 2019.

8. Aksan A, Isik H, Radeke HH, Dignass A, Stein J. Systematic review with network meta-analysis: comparative efficacy and tolerability of different intravenous iron formulations for the treatment of iron deficiency anaemia in patients with inflammatory bowel disease. Aliment Pharmacol Ther. 2017;45:1303-18. https://doi.org/10.1111/ apt.14043.

9. Bager P, Hvas CL, Dahlerup JF. Drug-specific hypophosphatemia and hypersensitivity reactions following different intravenous iron infusions. Br J Clin Pharmacol. 2017;83:1118-25. https://doi. org/10.1111/bcp.13189.

10. Mulder MB, van den Hoek, Hester L, Birnie E, van Tilburg, Antonie J P, Westerman EM. Comparison of hypersensitivity reactions of intravenous iron: iron isomaltoside-1000 $\left(\right.$ Monofer $^{\circledR}$ ) versus ferric carboxy-maltose $\left(\right.$ Ferinject $\left.^{\circledR}\right)$. A single center, cohort study. Br J Clin Pharmacol. 2018. https://doi.org/10.1111/bcp.13805.

11. FDA. Statement from FDA Commissioner Scott Gottlieb, M.D., on FDA's new strategic framework to advance use of real-world evidence to support development of drugs and biologics. 2018. https:// 
www.fda.gov/NewsEvents/Newsroom/PressAnnouncements/ucm62 7760.htm. Accessed 4 Mar 2019.

12. Auerbach M, Lykke LL. A single infusion of iron isomaltoside 1000 allows a more rapid hemoglobin increment than multiple doses of iron sucrose with a similar safety profile in patients with iron deficiency anemia. Blood. 2018;132:2334. https://doi.org/10.1182/blood -2018-99-110199.

13. Kalra PA, Bhandari S. Safety of intravenous iron use in chronic kidney disease. Curr Opin Nephrol Hypertens. 2016;25:529-35. https ://doi.org/10.1097/MNH.0000000000000263.
14. Wolf M. Effects of iron isomaltoside versus ferric carboxymaltose on hormonal control of phosphate homeostasis: the PHOSPHARE-IDA04/05 randomized controlled trials. Online abstract to the 101st Annual Meeting and Expo of the Endocrine Society. 23-26 March 2019. New Orleans, Louisiana, USA. 